\title{
A Preliminary Study of the Complement Component Iq Levels in Predicting the Efficacy of Combined Immunotherapy in Patients with Lung Cancer
}

\author{
Daoming Zhang' \\ Yuan $\mathrm{Li}^{\prime}$ \\ Haoyue $\mathrm{Li}^{2}$ \\ Tian Tang' \\ Yongfa Zheng' \\ Xufeng Guo' \\ Ximing $\mathrm{Xu}^{\mathrm{I}}$ \\ 'Cancer Center, Renmin Hospital of \\ Wuhan University, Wuhan, People's \\ Republic of China; ${ }^{2}$ Department of \\ Cardiology, Renmin Hospital of Wuhan \\ University, Wuhan, People's Republic of \\ China
}

\begin{abstract}
Objective: To evaluate the value of serum complement component 1q (C1q) levels in predicting the efficacy of combined immunotherapy in patients with lung cancer.

Methods: A total of 42 patients with lung cancer who received combined immunotherapy in the cancer center of Renmin Hospital of Wuhan University were included in this study. The clinical data of serum $\mathrm{Clq}$ and lactate dehydrogenase (LDH) levels before and three weeks after immunotherapy were collected.

Results: Response evaluation showed that the number of patients with complete response (CR), partial response (PR), stable disease (SD), and progressive disease (PD) was $0(0 \%)$, $26(61.9 \%), 14(33.3 \%)$, and 2 (4.8\%), respectively. The CR/PR group (patients with CR or $\mathrm{PR})$ showed higher $p \mathrm{C} 1 \mathrm{q}(\mathrm{C} 1 \mathrm{q}$ level before immunotherapy) and $i \mathrm{C} 1 \mathrm{q}(\mathrm{C} 1 \mathrm{q}$ level 3 weeks after immunotherapy) than the SD/PD group (patients with SD or PD). The LDH reduction (96.2\%) and C1q increment (84.6\%) in the CR/PR group 3 weeks after immunotherapy were higher than those of the SD/PD group, and the differences were statistically significant. Logistic regression analysis indicated that $p \mathrm{Clq}, i \mathrm{C} 1 \mathrm{q}$, and $\mathrm{LDH}$ level trends 3 weeks after the treatment were significantly correlated to the efficacy of combined immunotherapy with odds ratios of $8.185,5.500$, and 0.031 , respectively.

Conclusion: High $\mathrm{C} 1 \mathrm{q}$ levels before immunotherapy and increased $\mathrm{C} 1 \mathrm{q}$ levels and decreased LDH levels 3 weeks afterward suggest good therapeutic effects of combined immunotherapy in patients with lung cancer. Serum C1q levels have certain clinical significance in predicting the efficacy of combined immunotherapy.
\end{abstract}

Keywords: immunotherapy, efficacy prediction, complement component 1q

\section{Introduction}

In recent years, immunotherapy has achieved encouraging results in the treatment of lung cancer. Compared with traditional chemotherapy, immunotherapy has advantages of programmed cell death protein 1 (PD-1) inhibitors, programmed cell death ligand-1 (PD-L1) inhibitors, and cytotoxic T-lymphocyte-associated protein 4 (CTLA-4) inhibitors. With the success of domestically developed drugs and lowered prices, immunotherapy will be widely applied in lung cancer treatment.

Since the efficacy of single-agent immunotherapy is unsatisfactory, combined immunotherapy is often used in clinical practice. As it is important to screen out patients susceptible to immunotherapy, many studies have been devoted to finding predictors of immunotherapy efficacy. Multiple related markers such as PD-L1 and
Cancer Center, Renmin Hospital of Wuhan University, 238 Jiefang Road, Wuhan, 430060, People's Republic of China

Tel +86- I3707 I2065 I

Email doctorxul20@aliyun.com 
tumor mutational burden (TMB) have been widely applied in predicting the efficacy of immunotherapy for various tumors. Other predictors include tumor-infiltrating lymphocytes (TILs), ${ }^{1}$ DNA damage repair-related genes, ${ }^{2}$ and neutrophil to lymphocyte ratio (NLR). ${ }^{3}$ However, most of the existing studies are focused on predicting the efficacy of single immunotherapy. Therefore, finding the predictors for combined immunotherapy can guide clinical practice.

Mainly found in animal body fluids and on the cell surface, complements are small proteins that acquire biological activity after activation and mediate inflammation and immune response. ${ }^{4}$ The role of complements in tumor immunotherapy is attracting increasing attention from scientists. As an important component of the complement system, C1q initiates the classical pathway of the complement system when activated. In this study, a retrospective analysis was conducted on patients with lung cancer who received combined immunotherapy in our center from 2019 to 2020 to evaluate the value of serum C1q levels in predicting immunotherapy efficacy.

\section{Materials and Methods Study Population}

Clinical data were collected from the patients with lung cancer who received combined immunotherapy in the cancer center of our hospital from 2019 to 2020. The inclusion criteria were: (1) Patients whose medical data were complete with malignant lung tumors confirmed by pathological diagnosis and observed measurable lesions; (2) Stage III or stage IV patients according to the TNM stage classification for lung cancer; (3) Patients receiving immune checkpoint inhibitor (ICI) monotherapy or combined immunotherapy; (4) Patients receiving immunotherapy for more than 3 cycles. The exclusion criteria were: (1) Patients receiving immunotherapy for less than 3 cycles; (2) Patients with other concomitant malignant tumors. The collected data included the patient's age, gender, the Eastern Cooperative Oncology Group performance status (ECOG PS) score, pathological type, clinical stage, metastasis site, previous treatment history, combined immunotherapy, C1q levels, and LDH levels before and three weeks after the first immunotherapy.

This retrospective study was approved by the Ethics Committee of Renmin Hospital. Patients' identifiable data were anonymized, and the requirement for informed consent was waived due to the retrospective nature of the study. All patient data were confidential.

\section{Efficacy Evaluation}

The response evaluation was conducted following the Response Evaluation Criteria in Solid Tumors (RECIST 1.1). ${ }^{5}$ The evaluation criteria included complete response (CR), partial response (PR), stable disease (SD), and progressive disease (PD).

\section{Statistical Analysis}

The collected data were processed on IBM SPSS Statistics 23. Categorical data were expressed as rates, and measurement data were expressed as $\mathrm{x} \pm \mathrm{s}$, using the $\chi^{2}$ test or Fisher's exact test. Binary logistic regression was used for multivariate analysis. The cut-off $\mathrm{C} 1 \mathrm{q}$ value was determined by receiver operating characteristic (ROC) curves analysis. $P<0.05$ was considered statistically significant.

\section{Results}

\section{Characteristics of Patients}

The 42 patients in this study included 32 males and 10 females. In terms of pathological types, 21 patients were with adenocarcinoma, 14 were with squamous cell carcinoma, and 7 were with small cell carcinoma. The ICIs included Pembrolizumab (13), Camrelizumab (9), Sintilimab (8), Tislelizumab (10), and Atezolizumab (2). In terms of the treatment plans, 7 patients received immunotherapy alone, 20 received immunotherapy combined with chemotherapy, 1 received combined anti-angiogenic therapy, 3 received combined radiotherapy, and 11 received combined chemotherapy along with antiangiogenic therapy (Table 1).

\section{Response Evaluation Results}

After immunotherapy, imaging examinations were performed on the patients according to RECIST 1.1 for response evaluation. The number of patients with $\mathrm{CR}$, PR, SD, and PD was 0 (0\%), 26 (61.9\%), 14 (33.3\%), and $2(4.8 \%)$, respectively (Table 2 ).

\section{Comparison Among the Patients}

The patients were classified into the $\mathrm{CR} / \mathrm{PR}$ group and the $\mathrm{SD} / \mathrm{PD}$ group according to the response evaluation results. There was no statistically significant difference between the two groups in terms of gender, age, pathological type, Ki-67 index, tumor stage, history of chemotherapy, 
Table I Baseline Characteristics of the Patients

\begin{tabular}{|c|c|c|}
\hline Characteristics & No. & $\%$ \\
\hline \multicolumn{3}{|l|}{ Sex } \\
\hline Male & 32 & $76.2 \%$ \\
\hline Female & 10 & $23.8 \%$ \\
\hline \multicolumn{3}{|l|}{ Age } \\
\hline$<60$ & 15 & $35.7 \%$ \\
\hline$\geq 60$ & 27 & $64.3 \%$ \\
\hline \multicolumn{3}{|l|}{ Pathology } \\
\hline Adenocarcinoma & 21 & $50.0 \%$ \\
\hline Squamous cell carcinoma & 14 & $33.3 \%$ \\
\hline Small cell carcinoma & 7 & $16.7 \%$ \\
\hline \multicolumn{3}{|l|}{ Ki-67 } \\
\hline$<50 \%$ & 21 & $50.0 \%$ \\
\hline$\geq 50 \%$ & 21 & $50.0 \%$ \\
\hline \multicolumn{3}{|l|}{ Stage } \\
\hline III & 11 & $26.2 \%$ \\
\hline IV & 31 & $73.8 \%$ \\
\hline \multicolumn{3}{|l|}{ Immunotherapy drug } \\
\hline Pembrolizumab & 13 & $31.0 \%$ \\
\hline Camrelizumab & 9 & $21.4 \%$ \\
\hline Sintilimab & 8 & $19.0 \%$ \\
\hline Tislelizumab & 10 & $23.8 \%$ \\
\hline Atezolizumab & 2 & $4.8 \%$ \\
\hline \multicolumn{3}{|l|}{ Line of treatment } \\
\hline I & 12 & $28.6 \%$ \\
\hline 2 & 17 & $40.5 \%$ \\
\hline$\geq 3$ & 13 & $31.0 \%$ \\
\hline \multicolumn{3}{|l|}{ Combination therapy } \\
\hline $\mathrm{ICl}$ & 7 & $16.7 \%$ \\
\hline $\mathrm{ICl}+$ Chemotherapy & 20 & $47.6 \%$ \\
\hline $\mathrm{ICl}+$ Anti-angiogenic therapy & 1 & $2.4 \%$ \\
\hline $\mathrm{ICl}+$ Radiotherapy & 3 & $7.1 \%$ \\
\hline $\mathrm{ICl}+$ Chemotherapy+ Anti-angiogenic therapy & 11 & $26.2 \%$ \\
\hline
\end{tabular}

Table 2 The Tumor Response Results

\begin{tabular}{|l|l|l|}
\hline Response & No. & $\%$ \\
\hline CR & 0 & $0 \%$ \\
PR & 26 & $61.9 \%$ \\
SD & 14 & $33.3 \%$ \\
PD & 2 & $4.8 \%$ \\
\hline
\end{tabular}

Abbreviations: $C R$, complete response; $P R$, partial response; $S D$, stable disease; $\mathrm{PD}$, progressive disease.

number of immunotherapy lines, $p \mathrm{LDH}$, and $i \mathrm{LDH}(P>$ 0.05). The $\mathrm{CR} / \mathrm{PR}$ group showed higher $p \mathrm{Clq}$ and $i \mathrm{C} 1 \mathrm{q}$ than the SD/PD group $(222.84 \pm 49.78 \mathrm{mg} / \mathrm{L}$ vs 176.63 $\pm 29.88 \mathrm{mg} / \mathrm{L}, 237.54 \pm 55.77 \mathrm{mg} / \mathrm{L}$ vs $178.09 \pm 33.69 \mathrm{mg} /$
L). The $\mathrm{LDH}$ reduction (96.2\%) and $\mathrm{Clq}$ increment $(84.6 \%)$ in the CR/PR group 3 weeks after immunotherapy were higher than those in the $\mathrm{SD} / \mathrm{PD}$ group, and the differences were statistically significant $(P<0.05$; Tables 3 and 4).

\section{Logistic Regression Analysis}

In the logistic regression analysis, $p \mathrm{Clq}(0=$ less than or equal to $200 \mathrm{mg} / \mathrm{L}, 1=$ more than $200 \mathrm{mg} / \mathrm{L}$ ), C1q trends 3 weeks after immunotherapy $(0=$ decrease, $1=$ increase $)$, and LDH trends $(0=$ decrease, $1=$ increase $)$ were the independent variables, whereas treatment efficacy $(0=\mathrm{PD} / \mathrm{SD}, 1=\mathrm{PR} / \mathrm{CR})$ was the dependent variable. The results showed that the $\mathrm{Clq}$ level before immunotherapy and the trends of $\mathrm{C} 1 \mathrm{q}$ and $\mathrm{LDH}$ 3 weeks afterward were significantly correlated to the efficacy of combined immunotherapy with odds ratios of 8.185 , 5.500, and 0.031, respectively (Table 5). Patients with high baseline C1q levels are more likely to obtain high ORR, and patients with increased C1q or decreased DHL after immunotherapy probably receive better efficacy.

\section{The Value of Baseline $\mathrm{Clq}$ in Predicting Combined Immunotherapy Efficacy}

The ROC curves between efficacy and baseline C1q levels are presented in Figure 1. The area under the curve is 0.787 , the $\mathrm{CI}$ is 0.652 to 0.922 , and the difference is statistically significant $(P<0.05)$. The cut-off value of baseline $\mathrm{C} 1 \mathrm{q}$ is $209.5 \mathrm{mg} / \mathrm{L}$, the corresponding sensitivity is $53.8 \%$, and the specificity is $93.7 \%$.

\section{Discussion}

The most widely used immunotherapies in the treatment of lung cancer are mainly based on ICIs. There are currently three ICIs, two anti-PD-1 inhibitors (nivolumab and pembrolizumab), and one anti-PD-1 inhibitor (atezolizumab) involved in treating patients with non-small cell lung cancer. ${ }^{6}$ Therapeutic options targeting CTLA-4 are also adopted in clinical practice, which suppress the antigenpresenting cells (APCs) by depleting immune-stimulating cytokines, producing immunosuppressive cytokines, and constitutively expressing CTLA-4. ${ }^{7}$ In addition, immunotherapies also include immunological interventions such as active immunotherapy (eg, Bacillus CalmetteGuérin, BCG) and adoptive cell transfer, which includes transfer factor (TF), tumor-infiltrating lymphocytes (TIL), dendritic cell-cytokine induced killer (DC-CIK), and antigen-specific cancer vaccines (melanoma-associated 
Table 3 Clinical Data Comparison Between the CR/PR Group and the SD/PD Group

\begin{tabular}{|c|c|c|c|c|}
\hline & SD/PD & CR/PR & $\chi^{2}$ & $P$ \\
\hline Sex & & & 0.020 & 0.887 \\
\hline Male & 12 & 20 & & \\
\hline Female & 4 & 6 & & \\
\hline Age & & & 3.240 & 0.072 \\
\hline$<60$ & 3 & 12 & & \\
\hline$\geq 60$ & 13 & 14 & & \\
\hline Pathology & & & 2.524 & 0.283 \\
\hline Adenocarcinoma & 10 & II & & \\
\hline Squamous cell carcinoma & 5 & 9 & & \\
\hline Small cell carcinoma & I & 6 & & \\
\hline $\mathrm{Ki}-67$ & & & 1.615 & 0.204 \\
\hline$<50 \%$ & 10 & 11 & & \\
\hline$\geq 50 \%$ & 6 & 15 & & \\
\hline Stage & & & 0.019 & 0.891 \\
\hline III & 4 & 7 & & \\
\hline IV & 12 & 19 & & \\
\hline History of chemotherapy & & & 0.740 & 0.390 \\
\hline No & 3 & 8 & & \\
\hline Yes & 13 & 18 & & \\
\hline Line of treatment & & & 0.185 & 0.912 \\
\hline I & 4 & 8 & & \\
\hline 2 & 7 & 10 & & \\
\hline$\geq 3$ & 5 & 8 & & \\
\hline Change trend of LDH & & & 14.994 & $0.00 I^{* *}$ \\
\hline Reduce & 7 & 25 & & \\
\hline Increase & 9 & I & & \\
\hline Change trend of $\mathrm{Clq}$ & & & 5.815 & $0.016 *$ \\
\hline Reduce & 8 & 4 & & \\
\hline Increase & 8 & 22 & & \\
\hline pLDH & & & 0.303 & 0.582 \\
\hline$\leq 230 \mathrm{U} / \mathrm{L}$ & 6 & 12 & & \\
\hline$>230 \mathrm{U} / \mathrm{L}$ & 10 & 14 & & \\
\hline$i \mathrm{LDH}$ & & & 1.265 & 0.261 \\
\hline$\leq 230 \mathrm{U} / \mathrm{L}$ & 7 & 16 & & \\
\hline$>230 \mathrm{U} / \mathrm{L}$ & 9 & 10 & & \\
\hline $\mathrm{pClq}$ & & & 8.636 & $0.003 * *$ \\
\hline$\leq 200 \mathrm{mg} / \mathrm{L}$ & 13 & 9 & & \\
\hline$>200 \mathrm{mg} / \mathrm{L}$ & 3 & 17 & & \\
\hline$i \mathrm{Clq}$ & & & 7.769 & $0.005^{* *}$ \\
\hline$\leq 200 \mathrm{mg} / \mathrm{L}$ & 12 & 8 & & \\
\hline$>200 \mathrm{mg} / \mathrm{L}$ & 4 & 18 & & \\
\hline
\end{tabular}

Notes: Calculated by Fisher's exact test. $* \mathrm{P}<0.05, * * \mathrm{P}<0.01$.

Abbreviations: $p \mathrm{LDH}, \mathrm{LDH}$ before treatment; iLDH, LDH three weeks after immunotherapy; $\mathrm{pClq}, \mathrm{Clq}$ before treatment; $i \mathrm{Clq}, \mathrm{Clq}$ three weeks after immunotherapy.
Table 4 Comparison of $\mathrm{LDH}$ and $\mathrm{Clq}$ Levels Between the Two Groups ( $x \pm s)$

\begin{tabular}{|l|l|l|l|}
\hline & CR+PR & SD+PD & $P$ \\
\hline$p L D H$ & $260.69 \pm 77.16$ & $248.31 \pm 45.12$ & 0.516 \\
$i \mathrm{LDH}$ & $230.62 \pm 61.99$ & $259.94 \pm 63.02$ & 0.147 \\
$i \mathrm{LDH}$ & $222.84 \pm 49.78$ & $176.63 \pm 29.88$ & $0.002^{* *}$ \\
$i \mathrm{Clq}$ & $237.54 \pm 55.77$ & $178.09 \pm 33.69$ & $0.000^{* *}$ \\
\hline
\end{tabular}

Notes: Values conformed to normal distribution. Results were presented as the mean $\pm S D$. $* P<0.05, * * P<0.01$.

Table 5 Multivariate Analysis of Risk Factors of Treatment Response

\begin{tabular}{|l|l|l|l|}
\hline Variable & $\boldsymbol{P}$ & $\mathbf{O R}$ & $\mathbf{9 5 \%} \mathbf{C l}$ \\
\hline$p \mathrm{LDH}$ & $0.006^{* *}$ & 8.185 & $1.839-36.424$ \\
Change trend of Clq & $0.021^{*}$ & 5.5 & $1.293-23.389$ \\
Change trend of LDH & $0.002^{* *}$ & 0.031 & $0.003-0.289$ \\
\hline
\end{tabular}

Notes: Binary logistic regression was used for multivariate analysis. ${ }^{*} \mathrm{P}<0.05$, $* * \mathrm{p}<0.01$.

antigen 3, MAGE-A3 and L-BLP25) ${ }^{8,9}$ Since not all cancer patients are susceptible to current treatments, combining with other anti-tumor therapies has become the mainstream idea in clinical practice. Randomized controlled trials have confirmed that adding ICIs to chemotherapy can improve patient prognosis. ${ }^{10}$ Mutations in proto-oncogenes and tumor suppressor genes probably affect the treatment response and survival of lung cancer

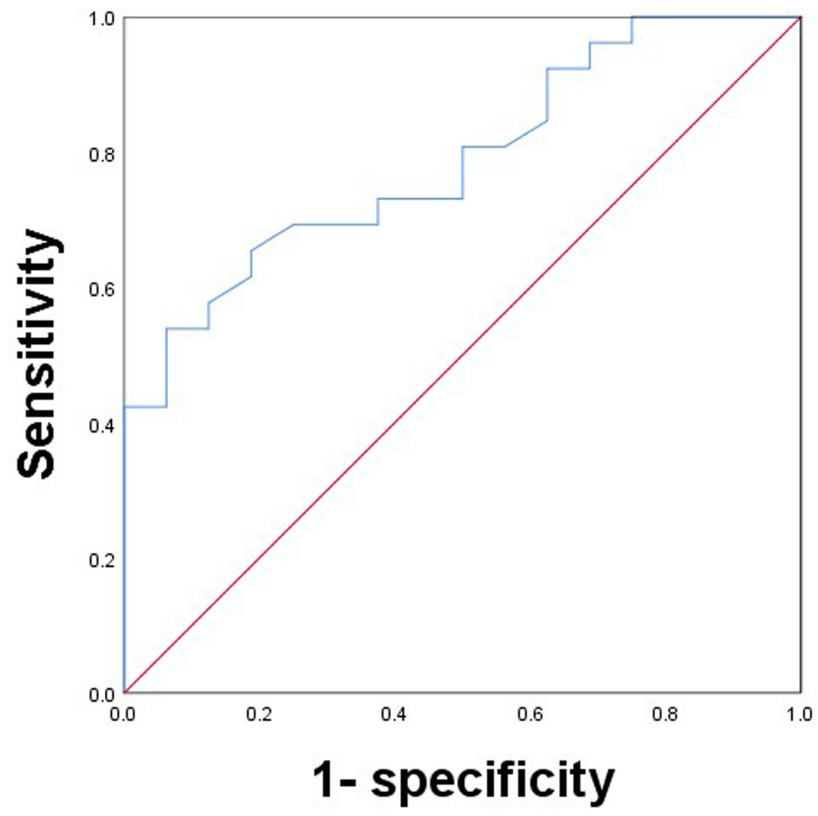

Figure I Multivariate analysis on the risk factors of treatment response. 
patients. Exploring the genetic background of different populations is of great significance to predict lung cancer patients' response to immunotherapies. ${ }^{11}$ In the application of ICIs, immune-related adverse events (irAEs) are the key monitoring items in clinical practice. The incidence of all grade irAEs is $22 \%$, and that of high-grade irAEs is $4 \%$. The most common orders are the endocrine system, skin, lungs, and gastrointestinal tract. Heart-related events have a high mortality rate and are nonspecific. Therefore, early identification of irAEs is crucial. ${ }^{12}$

Theoretically, upregulated expression of PD-L1 suggests a strong inhibitory effect of the PD-1/PD-L1 pathway, which indicate possible favorable effect of immune checkpoint inhibitors. Although early studies have confirmed the strong correlation between the two, PD-L1 has certain limitations as a predictor of pan-carcinoma. For example, research on the second-line treatment of renal cell carcinoma suggested that patients could benefit from nivolumab treatment regardless of whether the expression of PD-L1 was higher than $1 \%{ }^{13}$ In CheckMate 026, patients with non-small cell lung cancer receiving nivolumab treatment with PD-L1 expression $>5 \%$ showed unfavorable PFS and OS compared with patients receiving chemotherapy. ${ }^{14}$ In addition, PD-L1 is detected by immunohistochemistry in biopsy tissue. Different materials, antibodies, and automatic immunohistochemistry analysis systems all could lead to divergences in the results. In the meantime, the expression of PD-L1 in the tumor microenvironment may be correlated with efficacy. ${ }^{15}$ Finding and developing more treatment efficacy predictors and prediction models are still of great significance in clinical practice.

As part of the anti-tumor cytotoxicity and immune response, the activated complement system also promotes tumor development. On the one hand, complements have various regulatory effects, such as $\mathrm{C} 3 \mathrm{~b} / \mathrm{iC} 3 \mathrm{~b}$-mediated phagocytosis and TCC-mediated cytolysis. ${ }^{16}$ On the other hand, the excessive activation of complements also promotes tumor growth through the pro-inflammatory properties of the effector compounds. ${ }^{17}$ Cho et al reported that the local production and activation of complement effector compounds significantly promoted tumor growth. ${ }^{18}$

As the recognition subcomponent of the classical pathway of the complement system, $\mathrm{Clq}$ is responsible for eliminating immune complexes and invading pathogens. Upon recognition by $\mathrm{C} 1 \mathrm{r}$ and $\mathrm{C} 1 \mathrm{~s}$, the ligand triggers complement activation. ${ }^{19,20}$ Studies have found that C1q expression is upregulated in the microenvironment of various human tumors, which can promote and inhibit tumor development. $^{21,22}$ For example, in prostate cancer, C1q has been shown to induce apoptosis by activating tumor suppressor WOX $1,{ }^{23}$ while in malignant pleural mesothelioma, C1q promotes tumor cell adhesion, migration, and proliferation. $^{22}$ Studies have found that in clear cell renal cell carcinoma, C1q produced by tumor-associated macrophages, together with carcinoma cells expressing $\mathrm{C} 1 \mathrm{r}, \mathrm{C} 1 \mathrm{~s}$, $\mathrm{C} 4$, and $\mathrm{C} 3$, initiate $\mathrm{CP}$ activation and further affect the immunosuppressive microenvironment characterized by high expression of immune checkpoints (such as PD-1, lag3, PD-L1, and PD-L2), thereby promoting tumor progression. ${ }^{24}$ Therefore, we predict that the expression of Clq have a certain predictive value for the efficacy of immunotherapy.

According to preliminary clinical data of the CheckMate 017 study, the ORR of PD-1 inhibitors in second-line and later-line monotherapy was only $20 \%,{ }^{25}$ and the ORR in CheckMate 057 and KEYNOTE 010 was even lower. ${ }^{26,27}$ Therefore, combined immunotherapy is an effective way to improve the therapeutic effect. In this study, most patients were treated with ICIs combined with chemotherapy or anti-angiogenesis therapy. There was no statistically significant difference in treatment efficacy between patients of different gender, ages, pathological types, Ki-67 index, and the number of immune lines. Patients with higher $\mathrm{Clq}$ levels before treatment and higher $\mathrm{C} 1 \mathrm{q}$ levels three weeks afterward have higher ORR. In the meantime, patients with elevated $\mathrm{C} 1 \mathrm{q}$ levels after immunotherapy had better treatment efficacy. LDH is an inflammatory index related to tumor burden. Elevated LDH levels lead to lactic acid production, which acidifies the cell microenvironment and promotes tumor angiogenesis and the suppression of $\mathrm{T}$ cell immunity, thereby promoting the growth of tumor cells. ${ }^{28}$ Multiple studies have shown that initial LDH levels are significantly correlated with the efficacy of ICIs in advanced lung cancer patients. ${ }^{29,30}$ However, no correlation between $\mathrm{LDH}$ levels before treatment and treatment efficacy was found in this study, which was probably attributed to the small sample size. It is worth noting that patients with decreased LDH levels three weeks after treatment showed higher ORR, suggesting that the dynamic changes of the related biological indicators after receiving immunotherapy also had certain value for predicting therapeutic efficacy.

In summary, serum $\mathrm{C} 1 \mathrm{q}$ levels have a certain value for predicting the efficacy of combined immunotherapy in 
patients with advanced lung cancer. However, as a singlecenter retrospective study with a small sample, this study may be biased. The sample size will be expanded in the follow-up studies to explore the value of serum $\mathrm{Clq}$ levels in predicting clinical survival time. In the meantime, future research will also focus on the impact of the complement system on the immune mechanism of malignant tumors.

\section{Conclusion}

Serum C1q levels are easy to obtain during routine blood testing. High $\mathrm{C} 1 \mathrm{q}$ level before immunotherapy and increased $\mathrm{Clq}$ level and decreased LDH level three weeks afterward suggest good efficacy in combined immunotherapy. $\mathrm{C} 1 \mathrm{q} \geq 209.5 \mathrm{mg} / \mathrm{L}$ can probably serve as a cutoff for predicting the efficacy of combined immunotherapy in advanced lung cancer.

\section{Data Sharing Statement}

The datasets in this study can be obtained from the corresponding author according to reasonable requirements.

\section{Acknowledgments}

This study was supported by the National Natural Science Fund of China (Grant No. 31971166).

\section{Disclosure}

The authors declare no conflicts of interest regarding the publication of this paper.

\section{References}

1. Arora S, Velichinskii R, Lesh RW, et al. Existing and emerging biomarkers for immune checkpoint immunotherapy in solid tumors. Adv Ther. 2019;36:4. doi:10.1007/s12325-019-01051-z

2. Min YT, Seier K, Ostrovnaya I, et al. Alterations in DNA damage response and repair genes as potential marker of clinical benefit from PD-1/PD-L1 blockade in advanced urothelial cancers. J Clin Oncol. 2018;36(17):1685-1694. doi:10.1200/JCO.2017.75.7740

3. Ichiki Y, Taira A, Chikaishi Y, et al. Prognostic factors of advanced or postoperative recurrent non-small cell lung cancer targeted with immune check point inhibitors. J Thorac Dis. 2019;11(4):1117-1123. doi: $10.21037 /$ jtd.2019.04.41

4. Corrales L, Ajona D, Rafail S, et al. Anaphylatoxin C5a creates a favorable microenvironment for lung cancer progression. J Immunol. 2012;189(9):4674. doi:10.4049/jimmunol.1201654

5. Wolchok JD, Hoos A, O'Day S, et al. Guidelines for the evaluation of immune therapy activity in solid tumors: immune-related response criteria. Clin Cancer Res. 2009;15(23):7412-7420. doi:10.1158/10780432.CCR-09-1624

6. Tartarone A, Roviello G, Lerose R, et al. Anti-PD-1 versus anti-PD-L1 therapy in patients with pretreated advanced non-small-cell lung cancer: a meta-analysis. Future Oncol. 2019;15(20):2423-2433. doi:10. 2217/fon-2018-0868
7. Sobhani N, Tardiel-Cyril DR, Davtyan A, et al. CTLA-4 in regulatory $\mathrm{T}$ cells for cancer immunotherapy. Cancers. 2021;13(6):1440. doi:10.3390/cancers 13061440

8. Zhu J, Li R, Tiselius E, et al. Immunotherapy (excluding checkpoint inhibitors) for stage I to III non-small cell lung cancer treated with surgery or radiotherapy with curative intent. Cochrane Database Syst Rev. 2017;12(12):CD011300. doi:10.1002/14651858.CD011300.pub2

9. Mohsenzadegan M, Peng RW, Roudi R, et al. Dendritic cell/cytokine-induced killer cell-based immunotherapy in lung cancer: what we know and future landscape. $J$ Cell Physiol. 2020;235(1):74-86. doi: $10.1002 /$ jcp. 28977

10. Petrelli F, Ferrara R, Signorelli D, et al. Immune checkpoint inhibitors and chemotherapy in first-line NSCLC: a meta-analysis. Immunotherapy. 2021;13(7):621-631. doi:10.2217/imt-2020-0224

11. Fathi Z, Mousavi SAJ, Roudi R, et al. Distribution of KRAS, DDR2, and TP53 gene mutations in lung cancer: an analysis of Iranian patients. PLoS One. 2018;13(7):e0200633. doi:10.1371/journal.pone. 0200633

12. Sun X, Roudi R, Dai T, et al. Immune-related adverse events associated with programmed cell death protein-1 and programmed cell death ligand 1 inhibitors for non-small cell lung cancer: a PRISMA systematic review and meta-analysis. BMC Cancer. 2019;19(1):558. doi:10.1186/s12885-019-5701-6

13. Motzer RJ, Escudier B, Mcdermott DF, et al. Nivolumab versus everolimus in advanced renal-cell carcinoma. $N$ Engl $J$ Med. 2015;373(19):1803-1813. doi:10.1056/NEJMoa1510665

14. Carbone DP, Reck M, Paz-Ares L, et al. First-line nivolumab in stage IV or recurrent non-small-cell lung cancer. $N$ Engl J Med. 2017;376 (25):2415-2426. doi:10.1056/NEJMoa1613493

15. Georg. H. PD-L1 testing, fit for routine evaluation? From a pathologist's point of view. Memo. 2016;9(4):201-206. doi:10. 1007/s12254-016-0292-2

16. Wang SY, Weiner G. Complement and cellular cytotoxicity in antibody therapy of cancer. Expert Opin Biol Ther. 2008;8(6):759-768. doi:10.1517/14712598.8.6.759

17. Reis ES, Mastellos DC, Ricklin D, et al. Complement in cancer: untangling an intricate relationship. Nat Rev Immunol. 2018;18 (1):5-18. doi:10.1038/nri.2017.97

18. Cho MS, Vasquez HG, Rupaimoole R, et al. Autocrine effects of tumor-derived complement. Cell Rep. 2014;6(6):1085-1095. doi:10. 1016/j.celrep.2014.02.014

19. Lu J, Kishore U. C1 complex: an adaptable proteolytic module for complement and non-complement functions. Front Immunol. 2017;8:592. doi:10.3389/fimmu.2017.00592

20. Reid KKBM. C1q: structure, function, and receptors. Immunopharmacology. 2000;49(1-2):159-170. doi:10.1016/s01623109(00)80301-x

21. Bulla R, Tripodo C, Rami D, et al. C1q acts in the tumour microenvironment as a cancer-promoting factor independently of complement activation. Nat Commun. 2016;7(1):10346. doi:10.1038/ ncomms 10346

22. Agostinis C, Vidergar R, Belmonte B, et al. Complement protein C1q binds to hyaluronic acid in the malignant pleural mesothelioma microenvironment and promotes tumor growth. Front Immunol. 2017;8:1559. doi:10.3389/fimmu.2017.01559

23. Hong Q, Sze CI, Lin SR, et al. Complement C1q activates tumor suppressor WWOX to induce apoptosis in prostate cancer cells. PLoS One. 2009;4(6):e5755. doi:10.1371/journal.pone.0005755

24. Roumenina LT, Daugan MV, Noé R, et al. Tumor cells hijack macrophage-produced complement $\mathrm{C} 1 \mathrm{q}$ to promote tumor growth. Cancer Immunol Res. 2019;7(7):1091-1105. doi:10.1158/2326-6066. CIR-18-0891

25. Herbst RS, Baas P, Kim DW, et al. Pembrolizumab versus docetaxel for previously treated, PD-L1-positive, advanced non-small-cell lung cancer (KEYNOTE-010): a randomised controlled trial. Lancet. 2015;387(10027):1540-1550. doi:10.1016/S0140-6736(15)01281-7 
26. Borghaei H, Paz-Ares L, Horn L, et al. Nivolumab versus docetaxel in advanced nonsquamous non-small-cell lung cancer. $N$ Engl J Med. 2015:123-135. doi:10.1056/NEJMoa1507643

27. Brahmer J, Reckamp KL, Baas P, et al. Nivolumab versus docetaxel in advanced squamous-cell non-small-cell lung cancer. $N$ Engl J Med. 2015;373(2):123-135. doi:10.1056/NEJMoa1504627

28. Koukourakis MI, Giatromanolaki A, Sivridis E, et al. Lactate dehydrogenase-5 (LDH-5) overexpression in non-small-cell lung cancer tissues is linked to tumour hypoxia, angiogenic factor production and poor prognosis. Br J Cancer. 2003;89(5):877-885. doi:10.1038/ sj.bjc. 6601205
29. Mezquita L, Auclin E, Ferrara R, et al. Association of the lung immune prognostic index with immune checkpoint inhibitor outcomes in patients with advanced non-small cell lung cancer. JAMA Oncol. 2018;4(3):351-357. doi:10.1001/jamaoncol.2017.4771

30. Taniguchi Y, Tamiya A, Isa SI, et al. Predictive factors for poor progression-free survival in patients with non-small cell lung cancer treated with nivolumab. Anticancer Res. 2017;37(10):5857-5862. doi:10.21873/anticanres. 12030

\section{Publish your work in this journal}

Cancer Management and Research is an international, peer-reviewed open access journal focusing on cancer research and the optimal use of preventative and integrated treatment interventions to achieve improved outcomes, enhanced survival and quality of life for the cancer patient.
The manuscript management system is completely online and includes a very quick and fair peer-review system, which is all easy to use. Visit http://www.dovepress.com/testimonials.php to read real quotes from published authors. 